\title{
A Mixed Problem for Quasilinear Impulsive Hyperbolic Equations with Non Stationary Boundary and Transmission Conditions
}

\author{
Akbar B. Aliev' ${ }^{1}$ and Ulviya M. Mamedova ${ }^{2}$ \\ ${ }^{1}$ Azerbaijan Technical University, AZ 1073, Baki, Azerbaijan \\ ${ }^{2}$ Institute of Mathematics and Mechanics of NAS of Azerbaijan, AZ 1141, Baku, Azerbaijan \\ Correspondence should be addressed to Akbar B. Aliev, aliyevagil@yahoo.com \\ Received 10 March 2010; Revised 13 June 2010; Accepted 26 October 2010 \\ Academic Editor: Toka Diagana \\ Copyright (C) 2010 A. B. Aliev and U. M. Mamedova. This is an open access article distributed \\ under the Creative Commons Attribution License, which permits unrestricted use, distribution, \\ and reproduction in any medium, provided the original work is properly cited. \\ The initial-boundary value problem for a class of linear and nonlinear equations in Hilbert space \\ is considers. We prove the existence and uniqueness of solution of this problem. The results of \\ this investigation are applied to solvability of initial-boundary value problems for quasilinear \\ impulsive hyperbolic equations with non-stationary transmission and boundary conditions.
}

\section{Abstract Model Initial Boundary Value Problem with Non Stationary Boundary and Transmission Conditions for the Impulsive Linear Hyperbolic Equations}

In paper [1] there is given an abstract scheme of investigation of mixed problems for hyperbolic equations with non stationary boundary conditions. In this direction, some results were obtained in [2].

In this paper, we offer the analogues abstract model of investigation of mixed problem with non stationary boundary and transmission conditions for impulsive linear and semilinear hyperbolic equations.

\subsection{Statement of the Problem and Main Theorem}

Let $H^{i}, H_{0}^{i}, X_{v}^{i}, Y_{\mu}^{j}\left(v=1,2, \ldots, s_{i} ; i=1,2, \ldots, m ; \mu=1,2, \ldots, r_{j} ; j=1,2, \ldots, m\right)$ be Hilbert Spaces. Consider the following abstract initial-boundary value problem: 


$$
\begin{gathered}
\ddot{u}_{i}(t)+A_{i}(t) u_{i}(t)=f_{i}(t), \quad \text { (hyperbolic equations), } \\
B_{i v} \ddot{u}_{i}(t)+\sum_{k=1}^{m} C_{k v}^{i}(t) u_{k}(t)=g_{i v}(t), \quad(\text { non stationary boundary and transmission conditions), } \\
\sum_{k=1}^{m} D_{k \mu}^{i} u_{k}(t)=0, \quad(\text { stationary boundary and transmission conditions), } \\
u_{i}(0)=u_{i}^{0}, \quad \dot{u}_{i}(0)=u_{i}^{1}, \quad \text { (initial conditions), }
\end{gathered}
$$

where $t \in[0, T], \ddot{u}_{i}=d^{2} u_{i} / d t^{2}, \dot{u}_{i}=d u_{i} / d t, A_{i}(t)$ are the linear closed operators in $H^{i} ; B_{i v}$ are the linear operators from $H^{i}$ to $X_{v}^{i} ; C_{k v}^{i}(t)$ are the linear operators from $H^{k}$ to $X_{v}^{i} ; D_{k \mu}^{j}$ are the linear operators from $H^{k}$ to $Y_{\mu}^{j} ; v=1, \ldots, s_{i}, i=1, \ldots, m, \mu=1, \ldots, r_{j}, j=1, \ldots, m, k=1, \ldots, m$. We will investigate this problem under the following conditions.

(i) Let $H_{0}^{i} \subset H^{i}$, and let $H_{0}^{i}$ be densely in $H^{i}$ and continuously imbedded into it, $i=$ $1,2, \ldots, m$.

In the Hilbert space $H^{i}$, it was defined the system of the inner products $(\cdot, \cdot)_{H^{i}(t)}$, which generate uniform equivalent norms, that is,

$$
\begin{gathered}
c_{1}^{-1}\|u\|_{H^{i}}^{2} \leq\|u\|_{H^{i}(t)}^{2} \leq c_{1}\|u\|_{H^{i}}^{2}, \quad c_{1}>0, \\
\|u\|_{H^{i}(t)}^{2}=(u, u)_{H^{i}(t)}, \quad t \in[0, T], i=1,2, \ldots, m .
\end{gathered}
$$

For each $u \in H^{i}$, the function $t \rightarrow\|u\|_{H^{i}(t)}^{2}:[0, T] \rightarrow R_{+}$is continuously differentiable, $i=1,2, \ldots, m$.

In the Hilbert space $X_{v}^{i}$, it was defined the system of the inner products $(\cdot, \cdot)_{X_{v_{i}}}$, which generate uniform equivalent norms, that is,

$$
\begin{gathered}
c_{2}^{-1}\|v\|_{X_{v}^{i}}^{2} \leq\|v\|_{X_{v}^{i}(t)}^{2} \leq c_{2}\|v\|_{X_{v}^{i}}^{2}, \quad c_{2}>0 \\
\|v\|_{X_{v}^{i}(t)}^{2}=(v, v)_{X_{v}^{i}(t)}, \quad t \in[0, T], v=1,2, \ldots, s_{i}, i=1,2, \ldots, m .
\end{gathered}
$$

For each $v \in X_{v}^{i}$, the function $t \rightarrow\|v\|_{X_{v}^{i}(t)}^{2}:[0, T] \rightarrow R_{+}$is continuously differentiable.

(ii) For each $t \in[0, T]$ and $i=1,2, \ldots, m, A_{i}(t)$ is a linear closed operator in $H^{i}$ whose domain is $H_{0}^{i} ; A_{i}(t)$ acts boundedly from $H_{0}^{i}$ to $H^{i} ; A_{i}(t)$ is strongly continuously differentiable.

(iii) The linear operators $B_{i v}$, that act from $H_{1 / 2}^{i}$ to $X_{v}^{i}$, bounded, where $H_{1 / 2}^{i}=$ $\left[H_{0}^{i}, H^{i}\right]_{1 / 2}$ is interpolation space between $H_{0}^{i}$ and $H^{i}$ of order $1 / 2\left(v=1, \ldots, s_{i}, i=\right.$ $1, \ldots, m)$ (see [3]).

(iv) For each $t \in[0, T]$, the linear operators $C_{k v}^{i}(t)$, that act from $H^{k}$ to $X_{v}^{i}$, are bounded; $C_{k v}^{i}(t)$ is strongly continuously differentiable $\left(v=1, \ldots, s_{i}, i=1, \ldots, m\right.$; $k=1, \ldots, m)$. 
(v) The linear operators $D_{k \mu^{\prime}}^{j}$ from $H_{1 / 2}^{k}$ into $Y_{\mu}^{j}$, act boundedly $\left(\mu=1, \ldots, r_{j}, j=\right.$ $1, \ldots, m ; k=1, \ldots, m)$.

Let us introduce the following designations:

$$
\begin{aligned}
& \widehat{H}=H^{1} \oplus \cdots \oplus H^{m}, \\
& \widehat{H}_{0}=\left\{\bar{u}: \bar{u}=\left(u_{1}, \ldots, u_{m}\right), u_{i} \in H_{0}^{i}, i=1, \ldots, m ;\right. \\
& \left.\sum_{k=1}^{m} D_{k \mu}^{j} u_{k}=0, \mu=1, \ldots, r_{j}, j=1, \ldots, m\right\}, \\
& \widehat{H}_{1 / 2}=\left\{\bar{u}: \bar{u}=\left(u_{1}, \ldots, u_{m}\right), u_{i} \in H_{1 / 2}^{i}, i=1, \ldots, m ;\right. \\
& \left.\sum_{k=1}^{m} D_{k \mu}^{j} u_{k}=0, \mu=1, \ldots, r_{j}, j=1, \ldots, m\right\}, \\
& \mathscr{\ell}_{1}=\left\{w: w=\left(w_{1}, \ldots, w_{m}\right), w_{i}=\left(u_{i}, B_{i 1} u_{i}, \ldots, B_{i s_{i}} u_{i}\right), i=1, \ldots, m\right. \text {, } \\
& \text { where } \left.\left(u_{1}, \ldots, u_{m}\right) \in \widehat{H}_{0}\right\} \text {, } \\
& \mathscr{H}^{i}=H^{i} \oplus X_{1}^{i} \oplus \cdots \oplus X_{s_{i}}^{i}, \quad \mathscr{H}=\bigoplus_{i=1}^{m} \mathscr{H}^{i}, \quad \mathscr{H}_{1 / 2}=\left[\mathscr{H}_{1}, \mathscr{H}\right]_{1 / 2} .
\end{aligned}
$$

From condition (v), it follows that the space $\widehat{H}_{1 / 2}$ with the norm

$$
\|\bar{u}\|_{\widehat{H}_{1 / 2}}=\sum_{i=1}^{m}\left\|u_{i}\right\|_{H_{1 / 2}^{i}}
$$

is a subspace of

$$
H_{1 / 2}=\left\{\bar{u}: \bar{u}=\left(u_{1}, \ldots, u_{m}\right), u_{i} \in H_{1 / 2}^{i}, i=1, \ldots, m\right\}=H_{1 / 2}^{1} \times \cdots \times H_{1 / 2}^{m} .
$$

(vi) Let the linear manifold $\widehat{H}_{0}$ be dense in $\widehat{H}_{1 / 2}$, and let linear manifold $\mathscr{H}_{1}$ be dense in th.

(vii) (Green's Identity). For arbitrary $\bar{u}, \bar{v} \in \widehat{H}_{0}$ and $t \in[0, T]$, the following identity is valid:

$$
\begin{aligned}
& \sum_{i=1}^{m}[\left.\left(A_{i}(t) u_{i}, v_{i}\right)_{H^{i}(t)}+\sum_{v=1}^{s_{i}}\left(\sum_{k=1}^{m} C_{k v}^{i}(t) u_{k}, B_{i v} v_{i}\right)_{X_{v}^{i}(t)}\right] \\
&=\sum_{i=1}^{m}\left[\left(u_{i}, A_{i}(t) v_{i}\right)_{H^{i}(t)}+\sum_{v=1}^{s_{i}}\left(B_{i v} u_{i}, \sum_{k=1}^{m} C_{k v}^{i}(t) v_{k}\right)_{X_{v}^{i}(t)}\right] .
\end{aligned}
$$


(viii) For all $\bar{u}=\left(u_{1}, \ldots, u_{m}\right) \in \widehat{H}_{0}$, the following inequality is fulfilled:

$$
\begin{aligned}
& c_{1} \sum_{i=1}^{m}\left(\left\|u_{i}\right\|_{H^{i}}^{2}+\sum_{v=1}^{s_{i}}\left\|B_{i v} u_{i}\right\|_{X_{v}^{i}}^{2}\right) \\
& \quad \leq \sum_{i=1}^{m}\left[\left(A_{i}(t) u_{i}, u_{i}\right)_{H^{i}(t)}+\sum_{v=1}^{s_{i}}\left(\sum_{k=1}^{m} C_{k v}^{i}(t) u_{k}, B_{i v} u_{i}\right)_{X_{v}^{i}(t)}\right] \leq c_{2} \sum_{i=1}^{m}\left\|u_{i}\right\|_{H_{1 / 2}^{i}}^{2}
\end{aligned}
$$

where $c_{1} \in R, c_{2}>0$.

(ix) For each $t \in[0, T]$, an operator pencil

$$
\begin{aligned}
\mathcal{L}^{t}(\lambda): \bar{u} & =\left(u_{1}, \ldots, u_{m}\right) \longrightarrow \mathcal{L}^{t}(\lambda) \bar{u} \\
& =\left(L_{10}^{t}(\lambda) \bar{u}, L_{11}^{t}(\lambda) \bar{u}, \ldots, L_{1 s_{1}}^{t}(\lambda) \bar{u}, \ldots, L_{m 0}^{t}(\lambda) \bar{u}, L_{m 1}^{t}(\lambda) \bar{u}, \ldots, L_{m s_{m}}^{t}(\lambda) \bar{u}\right),
\end{aligned}
$$

which acts boundedly from $\widehat{H}_{0}$ to $\mathscr{H}$, has a regular point $\lambda=\lambda_{0} \in R$, where

$$
\begin{gathered}
L_{i 0}^{t}(\lambda) \bar{u}=\lambda u_{i}+A_{i}(t) u_{i}, \quad i=1,2, \ldots, m, \\
L_{i v}^{t}(\lambda) \bar{u}=\lambda B_{i v} u_{i}+\sum_{k=1}^{m} C_{k v}^{i}(t) u_{k}, \quad v=1,2, \ldots, s_{i}, i=1,2, \ldots, m .
\end{gathered}
$$

(x) $u_{i}^{0} \in H_{0}^{i}, u_{i}^{1} \in H_{1 / 2}^{i}, \sum_{k=1}^{m} D_{k \mu}^{j} u_{k}^{0}=0, \sum_{k=1}^{m} D_{k \mu}^{j} u_{k}^{1}=0$

$$
\left(i=1,2, \ldots, m, \mu=1,2, \ldots, r_{j}, j=1,2, \ldots, m\right) .
$$

(xi) $f_{i}(\cdot) \in W_{p}^{1}\left(0, T ; H^{i}\right), p \geq 1, i=1, \ldots, m$,

$$
g_{i v}(\cdot) \in W_{p}^{1}\left(0, T ; X_{v}^{i}\right), \quad p \geq 1, v=1, \ldots, s_{i}, i=1, \ldots, m
$$

Definition 1.1. The function $t \rightarrow\left(u_{1}(t), \ldots, u_{m}(t)\right)$ is called a solution of problem (1.1)-(1.2) if the function $t \rightarrow \bar{u}(t)=\left(u_{1}(t), \ldots, u_{m}(t)\right)$ from $[0, T]$ to $\widehat{H}_{0}$ is continuous, and the function

$$
t \longrightarrow\left(u_{1}(t), B_{11} u_{1}(t), \ldots, B_{1 s_{1}} u_{1}(t), \ldots, u_{m}(t), B_{m 1} u_{m}(t), \ldots, B_{m s_{m}} u_{m}(t)\right)
$$

from $[0, T]$ to $\mathscr{\ell}$ is twice continuously differentiable and (1.1)-(1.2) are satisfied.

Theorem 1.2. Let conditions (i)-(xi) are satisfied, then the problem (1.1)-(1.2) has a unique solution. 
Proof. We define the operator $\mathcal{A}(t)$ in the Hilbert space $\mathscr{t}$ in the following way:

$$
\begin{gathered}
D(\mathcal{A}(t))=\mathscr{L}_{1}, \\
\mathcal{A}(t) w=\left(A_{1}(t) u_{1}, \sum_{k=1}^{m} C_{k 1}^{1}(t) u_{k}, \ldots, \sum_{k=1}^{m} C_{k s_{1}}^{1}(t) u_{k}, \ldots, A(t)_{m} u_{m},\right. \\
\left.\sum_{k=1}^{m} C_{k 1}^{m}(t) u_{k}, \ldots, \sum_{k=1}^{m} C_{k s_{m}}^{m}(t) u_{k}\right), \quad t \in[0, T], w \in \mathscr{H}_{1} .
\end{gathered}
$$

Then the problem (1.1)-(1.2) is represented as the Cauchy problem

$$
\begin{gathered}
\ddot{w}+\mathscr{A}(t) w=\Phi(t), \\
w(0)=w^{0}, \quad \dot{w}(0)=w^{1},
\end{gathered}
$$

where $w(t)=\left(u_{1}(t), B_{11} u_{1}(t), \ldots, B_{1 s_{1}} u_{1}(t), \ldots, u_{m}(t), B_{m 1} u_{m}(t), \ldots, B_{m s_{m}} u_{m}(t)\right)$,

$$
\begin{gathered}
\Phi(t)=\left(f_{1}(t), g_{11}(t), \ldots, g_{1 s_{1}}(t), \ldots, f_{m}(t), g_{m 1}(t), \ldots, g_{m s_{m}}(t)\right), \\
w^{0}=\left(u_{1}^{0}, B_{11} u_{1}^{0}, \ldots, B_{1 s_{1}} u_{1}^{0}, \ldots, u_{m}^{0}, B_{m 1} u_{m}^{0}, \ldots, B_{m s_{m}} u_{m}^{0}\right), \\
w^{1}=\left(u_{1}^{1}, B_{11} u_{1}^{1}, \ldots, B_{1 s_{1}} u_{1}^{1}, \ldots, u_{m}^{1}, B_{m 1} u_{m}^{1}, \ldots, B_{m s_{m}} u_{m}^{1}\right) .
\end{gathered}
$$

It is obvious that if $\left(u_{1}(t), \ldots, u_{m}(t)\right)$ is the solution of problem (1.1)-(1.2), then $w(t)$ is the solution of the problem (1.16). On the contrary, if

$$
w(t) \in C^{2}([0, T] ; \mathscr{\ell}) \cap C^{1}\left([0, T] ;\left[\mathscr{\ell}_{1}, \mathscr{H}\right]_{1 / 2}\right) \cap C\left([0, T] ; \mathscr{\ell}_{1}\right)
$$

is the solution of problem (1.16), then $w(t)=\left(u_{1}(t), B_{11} u_{1}(t), \ldots, B_{1 s_{1}} u_{1}(t), \ldots, u_{m}(t)\right.$, $\left.B_{m 1} u_{m}(t), \ldots, B_{m s_{m}} u_{m}(t)\right)$ and $\left(u_{1}(t), \ldots, u_{m}(t)\right)$ is the solution of problem (1.1)-(1.2).

Let us define the system of inner product in Hilbert space $\mathscr{H}$ in the following way:

$$
\left(w^{1}, w^{2}\right)_{\mathscr{H}(t)}=\sum_{i=1}^{m}\left(w_{i}^{1}, w_{i}^{2}\right)_{H^{i}(t)}+\sum_{i=1}^{m} \sum_{v=1}^{s_{i}}\left(B_{i v} u_{i}^{1}, B_{i v} u_{i}^{2}\right)_{X_{v}^{i}(t)^{\prime}} \quad t \in[0, T]
$$

where $w^{l}=\left(w_{1}^{l}, \ldots, w_{m}^{l}\right), w_{i}^{l}=\left(u_{i^{\prime}}^{l}, B_{i 1} u_{i^{\prime}}^{l}, \ldots, B_{i s_{i}} u_{i}^{l}\right), i=1,2, \ldots, m,\left(u_{1}^{l}, \ldots, u_{m}^{l}\right) \in \widehat{H}_{0}, l=1,2$.

We denote space $\mathscr{d}$ with inner product (1.19) by $\mathscr{d}(t)$.

We will prove later the following auxiliary results.

Statement 1.3. There exists such $c_{3}>0$, that

$$
c_{3}^{-1}\|w\|_{\mathscr{d}}^{2} \leq\|w\|_{\mathscr{H}(t)}^{2} \leq c_{3}\|w\|_{\mathscr{d}}^{2}, \quad t \in[0, T]
$$


and the function $t \rightarrow\|w\|_{\mathscr{L}(t)}^{2}:[0, T] \rightarrow R_{+}$is continuously differentiable, where $\|w\|_{\mathscr{H}(t)}^{2}=$ $(w, w)_{\mathscr{L}(t)}$.

Statement 1.4. $\mathscr{A}(t)$ is a symmetric operator in $\mathscr{\ell}(t)$ for each $t \in[0, T]$.

Statement 1.5. $\mathscr{A}(t)$ has a regular point for each $t \in[0, T]$ in $R$.

$\mathscr{A}(t)$ is symmetric and $R(\mathscr{A}(t)+\lambda I)=\mathscr{H}(t)$, for some $\lambda \in R$; therefore, for each $t \in$ $[0, T], \mathscr{A}(t)$ is a selfadjoint operator in $\mathscr{H}(t)$ (see $[4$, chapter $\mathrm{x}]$ ).

Taking into account (viii) and Statement 1.3, we get

$$
\begin{aligned}
(\mathcal{A}(t) w, w)_{\mathscr{H}(t)} & =\sum_{i=1}^{m}\left[\left(A_{i}(t) u_{i}, u_{i}\right)_{H^{i}(t)}+\sum_{v=1}^{s_{i}}\left(\sum_{k=1}^{m} C_{k v}^{i}(t) u_{k}, B_{i v} u_{i}\right)_{X_{v}^{i}(t)}\right] \\
& \geq c_{1}\|w\|_{\mathscr{H}(t)}^{2},
\end{aligned}
$$

that is, $\mathscr{A}(t)$ is a lower semibounded selfadjoint operator in $\mathscr{\ell}(t)$.

Thus, the operator $\mathcal{A}_{0}(t)=\mathscr{A}(t)+\lambda_{0} I$ is selfadjoint and positive definite, where $\lambda_{0}>c_{1}$.

Problem (1.16) can be rewritten as

$$
\begin{gathered}
\ddot{w}(t)+\mathcal{A}_{0}(t) w(t)-\lambda_{0} w(t)=\mathcal{F}(t), \\
w(0)=w^{0}, \quad \dot{w}(0)=w^{1} .
\end{gathered}
$$

It is known that if $w^{0} \in \mathscr{H}_{1}$ and $w^{1} \in \mathscr{H}_{1 / 2}$, then the problem (1.22) has a unique solution $w \in C^{2}([0, T] ; \mathscr{\ell}) \cap C^{1}\left([0, T] ; \mathscr{\ell}_{1 / 2}\right) \cap C\left([0, T] ; \mathscr{\ell}_{1}\right)($ see $[5,6])$.

To complete the proof of the theorem, we need to show that $w^{0} \in \mathscr{H}_{1}$ and $w^{1} \in \mathscr{H}_{1 / 2}$.

By conditions of the theorem $u_{i}^{0} \in H_{0}^{i}, \sum_{k=1}^{m} D_{k \mu}^{j} u_{k}^{0}=0\left(i=1,2, \ldots, m ; \mu=1,2, \ldots, r_{j}\right.$, $j=1,2, \ldots, m)$ and $B_{i v}$ are bounded operators from $H_{1 / 2}^{i}$ to $X_{v}^{i}, v=1,2, \ldots, s_{i}, i=1,2, \ldots, m$. Therefore,

$$
w^{0}=\left(u_{1}^{0}, B_{11} u_{1}^{0}, \ldots, B_{1 s_{1}} u_{1}^{0}, \ldots, u_{m}^{0}, B_{m 1} u_{m}^{0}, \ldots, B_{m s_{m}} u_{m}^{0}\right) \in \mathscr{L}_{1} .
$$

On the other hand, $u_{i}^{1} \in H_{1 / 2}^{i}$ and $\sum_{k=1}^{m} D_{k \mu}^{j} u_{k}^{1}=0\left(i=1,2, \ldots, m, \mu=1,2, \ldots, r_{j}, j=\right.$ $1,2, \ldots, m)$, therefore, $B_{i v} u_{i}^{1} \in X_{v}^{i}\left(v=1,2, \ldots, s_{i}, i=1,2, \ldots, m\right)$. Consequently,

$$
\begin{gathered}
w^{1}=\left(u_{1}^{1}, B_{11} u_{1}^{1}, \ldots, B_{1 s_{1}} u_{1}^{1}, \ldots, u_{m}^{1}, B_{m 1} u_{m}^{1}, \ldots, B_{m s_{m}} u_{m}^{1}\right) \in \mathcal{\partial}, \\
\partial=\left\{w: w=\left(w_{1}, \ldots, w_{m}\right), w_{i}=\left(u_{i}, B_{i 1} u_{i}, \ldots, B_{i s_{i}} u_{i}\right), u_{i} \in H_{1 / 2}^{i},\right. \\
\left.\sum_{k=1}^{m} D_{k \mu}^{j} u_{k}=0, i=1, \ldots, m, \mu=1, \ldots, r_{j}, j=1, \ldots, m\right\} .
\end{gathered}
$$


From the definition of interpolation spaces (see [3, chapter 1$]$, [7, chapter 1$]$ ), we get the following inclusion:

$$
\mathscr{H}_{1} \subset \mathscr{H}_{1 / 2} \subset \widetilde{\mathscr{H}}_{1 / 2}=\bigoplus_{i=1}^{m}\left(H_{1 / 2}^{i} \oplus X_{1}^{i} \oplus \cdots \oplus X_{s_{i}}^{i}\right)
$$

By virtue of definition, the powers of positive selfadjoint operator (see [8, chapter 2], $\left[7\right.$, chapter 1]), we have that $D\left(\mathscr{A}_{0}^{1 / 2}(t)\right)=\mathscr{\ell}_{1 / 2}$ and

$$
c^{-1}\|w\|_{\mathscr{L}_{1 / 2}} \leq\left\|\mathscr{A}_{0}^{1 / 2}(t) w\right\|_{\mathscr{L}(t)} \leq c\|w\|_{\mathscr{L}_{1 / 2}} \quad c>0 .
$$

Assume that $w \in D\left(\mathscr{A}_{0}\right)=\mathscr{H}_{1}$, then

$$
\begin{aligned}
\left\|\mathcal{A}_{0}^{1 / 2}(t) w\right\|_{\mathscr{H}(t)}^{2}= & \left(\mathcal{A}_{0}(t) w, w\right)_{\mathscr{H}(t)} \\
= & \sum_{i=1}^{m}\left[\left(A_{i}(t) u_{i}, u_{i}\right)_{H^{i}(t)}+\sum_{v=1}^{s_{i}}\left(\sum_{k=1}^{m} C_{k v}^{i}(t) u_{k}, B_{i v} u_{i}\right)_{X_{v}^{i}(t)}\right] \\
& +\lambda_{0} \sum_{i=1}^{m}\left[\left(u_{i}, u_{i}\right)_{H^{i}(t)}+\sum_{v=1}^{s_{i}}\left(B_{i v} u_{i}, B_{i v} u_{i}\right)_{X_{v}^{i}(t)}\right] .
\end{aligned}
$$

By virtue of conditions (ii), (viii), (1.26), and (1.27), we get

$$
\|w\|_{\mathfrak{d}_{1 / 2}}^{2} \leq c \sum_{i=1}^{m}\left\|u_{i}\right\|_{H_{1 / 2}^{i}}^{2}
$$

Let $w^{1} \in 2$. By virtue of condition (vi), $\widehat{H}_{0}$ is dense in $\widehat{H}_{1 / 2}$; therefore, there exists a sequence $\bar{u}^{(p)}=\left(u_{1}^{(p)}, \ldots, u_{m}^{(p)}\right)$, such that $\bar{u}^{(p)} \in \widehat{H}_{0}$ and

$$
\left\|\bar{u}^{(p)}-\bar{u}^{1}\right\|_{H_{1 / 2}^{1} \oplus \cdots \oplus H_{1 / 2}^{m}} \longrightarrow 0, \quad \text { at } p \longrightarrow \infty
$$

Hence it follows, that

$$
\left\|\bar{u}^{(p)}-\bar{u}^{(q)}\right\|_{H_{1 / 2}^{1} \oplus \cdots \oplus H_{1 / 2}^{m}} \longrightarrow 0 \quad \text { at } p, q \longrightarrow \infty .
$$

Then from (1.28) and (1.30) it follows that $\left\{w^{(p)}\right\}$ is fundamental in $\mathscr{\ell}_{1 / 2}$, that is,

$$
\left\|w^{(p)}-w^{(q)}\right\|_{\mathscr{d}_{1 / 2}} \longrightarrow 0, \quad \text { at } p, q \longrightarrow \infty
$$

where $w^{(p)}=\left(u_{1}^{(p)}, B_{11} u_{1}^{(p)}, \ldots, B_{1 s_{1}} u_{1}^{(p)}, \ldots, u_{m}^{(p)}, B_{m 1} u_{m}^{(p)}, \ldots, B_{m s_{m}} u_{m}^{(p)}\right), p=1,2, \ldots$ 
Thus, there exists $\tilde{w} \in \mathscr{H}_{1 / 2}$ such that

$$
\left\|w^{(p)}-\tilde{w}\right\|_{\mathscr{e}_{1 / 2}} \longrightarrow 0, \quad \text { at } p \longrightarrow \infty .
$$

On the other hand, $\mathscr{l}_{1 / 2} \subset \widetilde{\mathscr{H}}_{1 / 2}$, therefore,

$$
\left\|w^{(p)}-\tilde{w}\right\|_{\widetilde{\mathscr{d}}_{1 / 2}} \longrightarrow 0, \quad \text { at } p \longrightarrow \infty .
$$

Hence,

$$
\left\|\bar{u}^{(p)}-\bar{u}\right\|_{H_{1 / 2}^{1} \oplus \cdots \oplus H_{1 / 2}^{m}} \longrightarrow 0, \quad \text { at } p \longrightarrow \infty,
$$

where $\bar{u}=\left(\widetilde{u}_{1}, \ldots, \widetilde{u}_{m}\right)$. From this, by virtue of $(1.29), \bar{u}=\bar{u}^{1}$, that is,

$$
\widetilde{w}=\left(u_{1}^{1}, B_{11} u_{1}^{1}, \ldots, B_{1 s_{1}} u_{1}^{1}, \ldots, u_{m}^{1}, B_{m 1} u_{m}^{1}, \ldots, B_{m s_{m}} u_{m}^{1}\right)=w^{1} .
$$

Thus, $w^{1} \in \mathscr{H}_{1 / 2}$. The theorem is proved.

\subsection{Proof of Auxiliary Results}

Validity of Statement 1.3 follows from condition (i), the Statement 1.4 from condition (vii).

Proof of Statement 3. Consider in Hilbert space $\mathcal{H}$ the equation

$$
\lambda w+\mathcal{A}(t) w=\mathcal{F}, \quad t \in[0, T]
$$

where $\mathcal{F}=\left(f_{1}, f_{11}, \ldots, f_{1 s_{1}}, \ldots, f_{m}, f_{m 1}, \ldots, f_{m s_{m}}\right) \in \mathscr{d}, \lambda \in R$. equations:

Equation (1.36) is equivalent to the following system of differential-operator

$$
\begin{gathered}
L_{i 0}^{t}(\lambda) \bar{u}=\lambda u_{i}+A_{i}(t) u_{i}=f_{i}, \quad t \in[0, T], i=1,2, \ldots, m, \\
L_{i v}^{t}(\lambda) \bar{u}=\lambda B_{i v} u_{i}+\sum_{k=1}^{m} C_{k v}^{i}(t) u_{k}=g_{i v}, \quad t \in[0, T], v=1,2, \ldots, s_{i}, i=1,2, \ldots, m, \\
\sum_{k=1}^{m} D_{k \mu}^{j} u_{k}=0, \quad \mu=1,2, \ldots, r_{j}, j=1,2, \ldots, m .
\end{gathered}
$$

By virtue of (ix), problem (1.37) has a solution $\bar{u}=\left(u_{1}, \ldots, u_{m}\right) \in \widehat{H}_{0}$ for some $\lambda \in R$. Thus, for each $t \in[0, T]$,

$$
R(\lambda I+\mathscr{A}(t))=\mathscr{H}(t),
$$

where $I$ is an identity operator in $\mathscr{H}(t)$, that is, $\mathbb{A}$ has a regular point. 


\section{Abstract Model of Initial Boundary Value Problem with Non Stationary Boundary and Transmission Conditions for the Impulsive Semilinear Hyperbolic Equations}

Consider the following initial boundary value problem:

$$
\begin{gathered}
\ddot{u}_{i}(t)+A_{i}(t) u_{i}(t)=f_{i}(t, \bar{u}(t), \dot{\bar{u}}(t)), \\
B_{i v} \ddot{u}_{i}(t)+\sum_{k=1}^{m} C_{k v}^{i}(t) u_{k}(t)=g_{i v}(t, \bar{u}(t), \ddot{\bar{u}}(t)), \\
\sum_{k=1}^{m} D_{k \mu}^{i} u_{k}(t)=0, \\
u_{i}(0)=u_{i}^{0}, \quad \dot{u}_{i}(0)=u_{i}^{1},
\end{gathered}
$$

where $t \in[0, T], v=1, \ldots, s_{i}, \mu=1, \ldots, r_{i}, i=1, \ldots, m, \dot{\bar{u}}=\left(u_{1}, \ldots, u_{m}\right), \ddot{\bar{u}}=\left(\dot{u}_{1}, \ldots, \dot{u}_{m}\right), A_{i}(t)$, $B_{i v}, C_{k v}^{i}(t)$ and $D_{k \mu}^{i}$ satisfy all conditions of Theorem 1.2.

Assume, that the nonlinear operators $f_{i}$ and $g_{i v}$ satisfy the following conditions.

$\left(\mathrm{xi}^{\prime}\right)$ Suppose that the nonlinear operators

$$
\begin{aligned}
& (t, \bar{u}, \dot{\bar{u}}) \longrightarrow f_{i}(t, \bar{u}, \dot{\bar{u}}):[0, T] \times\left(\bigoplus_{i=1}^{m} H_{1 / 2}^{i}\right) \times\left(\bigoplus_{i=1}^{m} H^{i}\right) \longrightarrow H^{i}, \\
& (t, \bar{u}, \dot{\bar{u}}) \longrightarrow g_{i v}(t, \bar{u}, \dot{\bar{u}}):[0, T] \times\left(\bigoplus_{i=1}^{m} H_{1 / 2}^{i}\right) \times\left(\bigoplus_{i=1}^{m} H^{i}\right) \longrightarrow X_{v}^{i}
\end{aligned}
$$

satisfy the local Lipschitz conditions in the following sense: for arbitrary $t_{1}, t_{2} \in$ $[0, T],\left(\bar{u}^{1}, \bar{v}^{1}\right),\left(\bar{u}^{2}, \bar{v}^{2}\right) \in \widehat{H}_{1 / 2} \times \widehat{H}$,

$$
\begin{aligned}
& \left\|f_{i}\left(t_{1}, \bar{u}^{1}, \bar{v}^{1}\right)-f_{i}\left(t_{2}, \bar{u}^{2}, \bar{v}^{2}\right)\right\|_{H^{i}} \\
& \quad \leq c_{i}(r)\left[\left|t_{1}-t_{2}\right|+\sum_{i=1}^{m}\left(\left\|u_{i}^{1}-u_{i}^{2}\right\|_{H_{1 / 2}^{i}}+\left\|v_{i}^{1}-v_{i}^{2}\right\|_{H^{i}}\right)\right], \\
& \left\|g_{i v}\left(t_{1}, \bar{u}^{1}, \bar{v}^{1}\right)-g_{i v}\left(t_{2}, \bar{u}^{2}, \bar{v}^{2}\right)\right\|_{X_{v}^{i}} \\
& \quad \leq c_{i v}(r)\left[\left|t_{1}-t_{2}\right|+\sum_{i=1}^{m}\left(\left\|u_{i}^{1}-u_{i}^{2}\right\|_{H_{1 / 2}^{i}}+\left\|v_{i}^{1}-v_{i}^{2}\right\|_{H^{i}}\right)\right],
\end{aligned}
$$

where $c_{i}(\cdot), c_{i v} \in C\left(R_{+}, R_{+}\right), v=1, \ldots, s_{i}, i=1, \ldots, m$,

$$
r=\sum_{i=1}^{m} \sum_{l=1}^{2}\left(\left\|u_{i}^{l}\right\|_{H_{1 / 2}^{i}}+\left\|v_{i}^{l}\right\|_{H^{i}}\right) .
$$


Theorem 2.1. Let conditions (i)-(x) and $\left(x i^{\prime}\right)$ be satisfied, then there exists $T^{\prime} \in(0, T]$, such that the problem (2.1) has a unique solution

$$
\bar{u}=\left(u_{1}, \ldots, u_{m}\right) \in C\left(\left[0, T^{\prime}\right), \widehat{H}_{0}\right) \cap C^{1}\left(\left[0, T^{\prime}\right), \widehat{H}_{1 / 2}\right) \cap C^{2}\left(\left[0, T^{\prime}\right), \widehat{H}\right) .
$$

Additionally, if

$$
E(t)=\sum_{i=1}^{m}\left[\left\|u_{i}(t)\right\|_{H_{1 / 2}^{i}}+\left\|\dot{u}_{i}(t)\right\|_{H^{i}}\right] \leq \varphi\left(\sum_{i=1}^{m}\left[\left\|u_{i}^{0}\right\|_{H_{1 / 2}^{i}}+\left\|u_{i}^{1}\right\|_{H^{i}}\right]\right), \quad t \in\left[0, T^{\prime}\right),
$$

where $\varphi(\cdot) \in C\left(R_{+}, R_{+}\right)$, then $T^{\prime}=T$. Otherwise, there exists $T_{0} \in(0, T)$, such that

$$
\lim _{t \rightarrow T_{0}-0} E(t)=+\infty
$$

In the Hilbert space $\mathscr{d}$, the problem (2.1) is represented as the Cauchy problem

$$
\begin{gathered}
\ddot{w}+\mathcal{A}_{0}(t) w=\mathcal{F}(t, w, \dot{w}), \\
w(0)=w^{0}, \quad \dot{w}(0)=w^{1},
\end{gathered}
$$

where $w=\left(u_{1}, B_{11} u_{1}, \ldots, B_{1 s_{1}} u_{1}, \ldots, u_{m}, B_{m 1} u_{m}, \ldots, B_{m s_{m}} u_{m}\right)$,

$$
\begin{gathered}
w^{0}=\left(u_{1}^{0}, B_{11} u_{1}^{0}, \ldots, B_{1 s_{1}} u_{1}^{0}, \ldots, u_{m}^{0}, B_{m 1} u_{m}^{0}, \ldots, B_{m s_{m}} u_{m}^{0}\right), \\
w^{1}=\left(u_{1}^{1}, B_{11} u_{1}^{1}, \ldots, B_{1 s_{1}} u_{1}^{1}, \ldots, u_{m}^{1}, B_{m 1} u_{m}^{1}, \ldots, B_{m s_{m}} u_{m}^{1}\right), \\
\mathcal{F}(t, w, \dot{w})=\lambda_{0} w+\mathcal{F}_{1}(t, w, \dot{w}) \\
\mathcal{F}_{1}(t, w, \dot{w})=\left(f_{1}(t, \bar{u}, \dot{\bar{u}}), g_{11}(t, \bar{u}, \dot{\bar{u}}), \ldots, g_{1 s_{1}}(t, \bar{u}, \dot{\bar{u}}), \ldots,\right. \\
\left.f_{m}(t, \bar{u}, \dot{\bar{u}}), g_{m 1}(t, \bar{u}, \dot{\bar{u}}), \ldots, g_{m s_{m}}(t, \bar{u}, \dot{\bar{u}})\right) .
\end{gathered}
$$

From ( $\left.\mathrm{xi}^{\prime}\right)$, it follows that, for arbitrary $t_{1}, t_{2} \in[0, T], w^{1}, w^{2} \in \mathscr{H}_{1 / 2}, z^{1}, z^{2} \in \mathscr{H}$,

$$
\left\|\mp\left(t_{1}, w^{1}, z^{1}\right)-\mp\left(t_{2}, w^{2}, z^{2}\right)\right\|_{\mathscr{\ell}} \leq c(r)\left[\left|t_{1}-t_{2}\right|+\left\|w^{1}-w^{2}\right\|_{\mathscr{L}_{1 / 2}}+\left\|z^{1}-z^{2}\right\|_{\mathscr{d}}\right]
$$

where $c(\cdot) \in C\left(R_{+}, R_{+}\right), \quad r=\sum_{l=1}^{2}\left(\left\|w^{l}\right\|_{\mathscr{d}_{1 / 2}}+\left\|z^{l}\right\|_{\mathscr{d}}\right)$. 
Thus, the nonlinear operator $\mathcal{F}$ satisfies the condition of local solvability of the Cauchy problem for the quasilinear hyperbolic equations in Hilbert space (see $[6,9])$. Taking this into account, the problem (2.8) has a unique solution

$$
w \in C^{2}\left(\left[0, T^{\prime}\right) ; \mathscr{l}\right) \cap C^{1}\left(\left[0, T^{\prime}\right) ; \mathscr{\ell}_{1 / 2}\right) \cap C\left(\left[0, T^{\prime}\right) ; \mathscr{\ell}_{1}\right) .
$$

\section{Initial Boundary Value Problem with Non Stationary Boundary and Transmission Condition for the Impulsive Semilinear Hyperbolic Equations}

Let $a_{1}<a_{2}<\cdots<a_{m+1}$. We consider in the domain $[0, T] \times \bigcup_{i=1}^{m}\left[a_{i}, a_{i+1}\right]$ the following mixed problem

$$
\begin{gathered}
\ddot{u}_{i}(t, x)-p_{i}(t) u_{i}^{\prime \prime}(t, x)=f_{i}\left(t, x, u_{i}(t, x), u_{i}^{\prime}(t, x), \dot{u}_{i}(t, x), \varphi_{i}(\bar{u}, \dot{\bar{u}})\right), \\
(t, x) \in[0, T] \times\left[a_{i}, a_{i+1}\right], \quad i=1,2, \ldots, m, \\
u_{i}\left(t, a_{i+1}\right)=u_{i+1}\left(t, a_{i+1}\right), \quad i=1,2, \ldots, m-1, t>0, \\
\ddot{u}_{1}\left(t, a_{1}\right)-q_{0}(t) u_{1}^{\prime}\left(t, a_{1}\right)=g_{0}\left(t, \psi_{0}(\bar{u}, \dot{\bar{u}})\right), \quad t>0, \\
\ddot{u}_{i}\left(t, a_{i+1}\right)+q_{i}(t)\left[u_{i}^{\prime}\left(t, a_{i+1}\right)-u_{i+1}^{\prime}\left(t, a_{i+1}\right)\right]=g_{i}\left(t, \psi_{i}(\bar{u}, \dot{\bar{u}})\right), \\
i=1,2, \ldots, m-1, t>0, \\
\ddot{u}_{m}\left(t, a_{m+1}\right)+q_{m}(t) u_{m}^{\prime}\left(t, a_{m+1}\right)=g_{m}\left(t, \psi_{m}(\bar{u}, \dot{\bar{u}})\right), \quad t>0, \\
u_{i}(0, x)=u_{i}^{0}(x), \quad \dot{u}_{i}(0, x)=u_{i}^{1}(x), \quad x \in\left[a_{i}, b_{i}\right], i=1,2, \ldots, m,
\end{gathered}
$$

where $\dot{u}_{i}=\partial u_{i} / \partial t, u_{i}^{\prime}=\partial u_{i} / \partial x, \ddot{u}_{i}=\partial^{2} u_{i} / \partial t^{2}, u_{i}^{\prime \prime}=\partial^{2} u_{i} / \partial x^{2}, \bar{u}=\left(u_{1}, \ldots, u_{m}\right), \dot{\bar{u}}=$ $\left(\dot{u}_{1}, \ldots, \dot{u}_{m}\right), p_{i}, q_{j}, f_{i}, g_{j}, u_{i}^{0}, u_{i}^{1}$ are some functions, $\varphi_{i}$ and $\psi_{j}$ are some functionals, which will be specified below, $i=1, \ldots, m, j=0,1, \ldots, m$.

Recently, differential equations with impulses are great interest because of the needs of modern technology, where impulsive automatic control systems and impulsive computing systems are very important and intensively develop broadening the scope of their applications in technical problems, heterogeneous by their physical nature and functional purpose (see [10, chapter 1]).

Assume that the following conditions are held:

$\left(1^{0}\right) p_{i}(t) \in C^{1}[0, T], q_{j}(t) \in C^{1}[0, T] ; p_{i}(t)>0, q_{j}(t)>0, t \in[0, T], i=1, \ldots, m, j=$ $0,1, \ldots, m$,

$\left(2^{0}\right) f_{i}(\cdot) \in C^{1}\left([0, T] \times\left[a_{i}, a_{i+1}\right] \times R^{4}\right), i=1,2, \ldots, m$ ，

$\left(3^{0}\right) g_{j}(\cdot) \in C^{1}([0, T], R), j=0,1, \ldots, m$, 
$\left(4^{0}\right) \varphi_{i}(\cdot)$ are nonlinear functionals acting from

$$
\bigoplus_{k=1}^{m}\left(W_{2}^{1}\left(a_{k}, a_{k+1}\right) \times L_{2}\left(a_{k}, a_{k+1}\right)\right)
$$

to $R$ and for arbitrary $\left(\bar{u}^{1}, \bar{v}^{1}\right),\left(\bar{u}^{2}, \bar{v}^{2}\right) \in \bigoplus_{k=1}^{m}\left(W_{2}^{1}\left(a_{k}, a_{k+1}\right) \times L_{2}\left(a_{k}, a_{k+1}\right)\right)$ the following inequality holds

$$
\begin{array}{r}
\left|\varphi_{i}\left(\bar{u}^{1}, \bar{v}^{1}\right)-\varphi_{i}\left(\bar{u}^{2}, \bar{v}^{2}\right)\right| \\
\leq c_{i}(r) \sum_{k=1}^{m}\left[\left\|u_{k}^{1}-u_{k}^{2}\right\|_{W_{2}^{1}\left(a_{k}, a_{k+1}\right)}+\left\|v_{k}^{1}-v_{k}^{2}\right\|_{L_{2}\left(a_{k}, a_{k+1}\right)}\right], \\
i=1,2, \ldots, m,
\end{array}
$$

where $r=\sum_{k=1}^{m}\left[\left\|u_{k}^{1}\right\|_{W_{2}^{1}\left(a_{k}, a_{k+1}\right)}+\left\|u_{k}^{2}\right\|_{W_{2}^{1}\left(a_{k}, a_{k+1}\right)}+\left\|v_{k}^{1}\right\|_{L_{2}\left(a_{k}, a_{k+1}\right)}+\left\|v_{k}^{1}\right\|_{L_{2}\left(a_{k}, a_{k+1}\right)}\right]$,

$$
c_{i}(\cdot) \in C\left(R_{+}, R_{+}\right), \quad R_{+}=[0, \infty), i=1,2, \ldots, m,
$$

$\left(5^{0}\right) \psi_{j}(\cdot)$ are nonlinear functionals acting from

$$
\bigoplus_{k=1}^{m}\left(W_{2}^{1}\left(a_{k}, a_{k+1}\right) \times L_{2}\left(a_{k}, a_{k+1}\right)\right)
$$

to $R$ and for arbitrary $\left(\bar{u}^{1}, \bar{v}^{1}\right),\left(\bar{u}^{2}, \bar{v}^{2}\right) \in \bigoplus_{k=1}^{m}\left(W_{2}^{1}\left(a_{k}, a_{k+1}\right) \times L_{2}\left(a_{k}, a_{k+1}\right)\right)$ the following inequality holds

$$
\left|\psi_{j}\left(\bar{u}^{1}, \bar{v}^{1}\right)-\psi_{j}\left(\bar{u}^{2}, \bar{v}^{2}\right)\right| \leq c_{j}(r) \sum_{k=1}^{m}\left[\left\|u_{k}^{1}-u_{k}^{2}\right\|_{W_{2}^{1}\left(a_{k}, a_{k+1}\right)}+\left\|v_{k}^{1}-v_{k}^{2}\right\|_{L_{2}\left(a_{k}, a_{k+1}\right)}\right]
$$

where $c_{j}(\cdot) \in C\left(R_{+}, R_{+}\right), j=0,1, \ldots, m$, and $r$-is defined as in (3.3),

$$
\left(6^{0}\right) u_{i}^{0} \in W_{2}^{2}\left(a_{i}, a_{i+1}\right), u_{i}^{1} \in W_{2}^{1}\left(a_{i}, a_{i+1}\right), i=1,2, \ldots, m \text {, where }
$$

$$
\begin{gathered}
u_{j}^{0}\left(a_{j+1}\right)=u_{j+1}^{0}\left(a_{j+1}\right), \\
u_{j}^{1}\left(a_{j+1}\right)=u_{j+1}^{1}\left(a_{j+1}\right), \quad j=1,2, \ldots, m-1 .
\end{gathered}
$$

By applying Theorem 2.1, we obtain the following result. 
Theorem 3.1. Let conditions $\left(1^{0}\right)-\left(6^{0}\right)$ be held, then there exists a $T^{\prime} \in(0, T]$, such that the problem (3.1) has a unique solution $\bar{u}=\left(u_{1}, \ldots, u_{m}\right)$, where

$$
\begin{gathered}
u_{i} \in C^{2}\left(\left[0, T^{\prime}\right) ; L_{2}\left(a_{i}, a_{i+1}\right)\right) \cap C^{1}\left(\left[0, T^{\prime}\right) ; W_{2}^{1}\left(a_{i}, a_{i+1}\right)\right) \cap C\left(\left[0, T^{\prime}\right) ; W_{2}^{2}\left(a_{i}, a_{i+1}\right)\right), \\
u_{i}\left(t, a_{i}\right), u_{i}\left(t, a_{i+1}\right) \in C^{2}\left(\left[0, T^{\prime}\right), R\right), \quad i=1,2, \ldots, m
\end{gathered}
$$

Proof. Let us denote $H^{i}=L_{2}\left(a_{i}, a_{i+1}\right), H_{0}^{i}=W_{2}^{2}\left(a_{i}, a_{i+1}\right), X_{v}^{i}=\mathbb{C}, Y_{\mu}^{j}=\mathbb{C}, v=1,2, \ldots, s_{i}, i=$ $1,2, \ldots, m, \mu=1,2, \ldots, r_{j}, j=1,2, \ldots, m$, where $s_{i}=2, r_{j}=1$.

In space $H^{i}$ and $X_{v}^{i}$ are defined the following inner products:

$$
\begin{gathered}
(u, v)_{H^{i}(t)}=p_{i}^{-1}(t) \int_{a_{i}}^{a_{i+1}} u \bar{v} d x, \\
\left(h_{1}, h_{2}\right)_{X_{1}^{1}(t)}=q_{0}^{-1}(t) h_{1} \bar{h}_{2}, \quad\left(h_{1}, h_{2}\right)_{X_{2}^{i}(t)}=q_{i}^{-1}(t) h_{1} \bar{h}_{2}, \\
h_{1}, h_{2} \in \mathbb{C}, \quad i=1,2, \ldots, m .
\end{gathered}
$$

From differentiability of the functions $p_{i}(t), i=1,2, \ldots, m$, and $q_{j}(t), j=0,1, \ldots, m$ it follows that the condition (i) is satisfied.

Let us define the following operators:

$$
\begin{aligned}
& A_{i}(t) u_{i}=-p_{i}(t) u_{i}^{\prime \prime}, u_{i} \in D\left(A_{i}(t)\right)=W_{2}^{2}\left(a_{i}, a_{i+1}\right), \\
& B_{11} u_{1}=u_{1}\left(a_{1}\right), B_{j 1}=0, B_{2 i} u_{i}=u_{i}\left(a_{i+1}\right), i=1,2, \ldots, m, j=2, \ldots, m, \\
& C_{11}^{1}(t) u_{1}=-q_{0}(t) u_{1}^{\prime}\left(a_{1}\right), C_{m 1}^{m}(t) u_{m}=q_{m}(t) u_{m}^{\prime}\left(a_{m+1}\right), \\
& C_{k 1}^{i}(t)=0 \text {, for all other } i, k \\
& C_{i 2}^{i}(t) u_{i}=q_{i}(t) u_{i}^{\prime}\left(a_{i+1}\right), i=1,2, \ldots, m, \\
& C_{j 2}^{j}(t) u_{j+1}=-q_{j}(t) u_{j+1}^{\prime}\left(a_{j+1}\right), j=1,2, \ldots, m-1, \\
& C_{k 2}^{i}(t)=0, \text { for all other } i, k, \\
& D_{i 1}^{i} u_{i}=-u_{i}\left(a_{i+1}\right), D_{i+1,1}^{i} u_{i+1}=u_{i+1}\left(a_{i+1}\right), i=1,2, \ldots, m-1, \\
& D_{k 1}^{i}=0, k \neq i, k \neq i+1 .
\end{aligned}
$$

We also define the nonlinear operators as follows:

$$
\begin{aligned}
& F_{i}(t, \bar{u}, \bar{v})=f_{i}\left(t, x, u_{i}(x), u_{i}^{\prime}(x), v_{i}(x), \varphi_{i}(\bar{u}, \bar{v})\right), i=1,2, \ldots, m, \\
& G_{11}(t, \bar{u}, \bar{v})=g_{0}\left(t, \psi_{0}(\bar{u}, \bar{v})\right) \\
& G_{i 2}(t, \bar{u}, \bar{v})=g_{i}\left(t, \psi_{i}(\bar{u}, \bar{v})\right), i=1,2, \ldots, m \\
& G_{i 1}(t, \bar{u}, \bar{v})=0, i=2,3, \ldots, m .
\end{aligned}
$$


It is easy to verify that linear operators $A_{i}(t), B_{i v}, C_{i v}^{k}(t)$, and $D_{i \mu}^{k}$ and the nonlinear operators $F_{i}, G_{i 1}$, and $G_{i 2}, i=1, \ldots, m$ satisfy the conditions of Theorem 2.1, and the problem (3.1) is represented as an abstract initial boundary-value problem in the following way:

$$
\begin{gathered}
\ddot{u}_{i}(t)+A_{i}(t) u_{i}(t)=F_{i}(t, \bar{u}, \dot{\bar{u}}), \\
B_{11} \ddot{u}_{1}(t)+C_{11}^{1}(t) u_{1}(t)=G_{0}(t, \bar{u}, \dot{\bar{u}}), \\
B_{i 2} \ddot{u}_{i}(t)+C_{i 2}^{i}(t) u_{i}(t)+C_{i+2,2}^{i}(t) u_{i}(t)=G_{i}(t, \bar{u}, \dot{\bar{u}}), \\
B_{m 2} \ddot{u}_{m}(t)+C_{m 2}^{m}(t) u_{m}(t)=G_{m}(t, \bar{u}, \dot{\bar{u}}), \\
D_{i 1}^{i} u_{i}+D_{i 1}^{i} u_{i+1}=0, \quad i=1,2, \ldots, m-1 .
\end{gathered}
$$

We will show that conditions of Theorem 2.1 are satisfied. Conditions (i) $-(\mathrm{v})$ follow immediately from definitions of spaces $H^{i}, X_{v}^{i}$, and $Y_{\mu}^{j}$ and operators $A_{i}(t), B_{i v}, C_{k v}^{i}(t)$, and $D_{k \mu^{\prime}}^{j}$ and traces theorems (see [3, chapter 2]), where $k=1,2, \ldots, m ; v=1,2, \ldots, s_{i}$; $i=1,2, \ldots, m ; \mu=1,2, \ldots, r_{j} ; j=1,2, \ldots, m$.

The linear manifolds $\widehat{H}_{0}$ and $\mathscr{H}_{1}$ are defined in the following way:

$$
\begin{gathered}
\widehat{H}_{0}=\left\{\bar{u}, \bar{u}=\left(u_{1}, \ldots, u_{m}\right), u_{i} \in W_{2}^{2}\left(a_{i}, a_{i+1}\right), i=1, \ldots, m,\right. \\
\left.u_{j}\left(a_{j+1}\right)=u_{j+1}\left(a_{j+1}\right), j=1, \ldots, m-1\right\}, \\
\mathscr{H}_{1}=\left\{w, w=\left(w_{1}, \ldots, w_{m}\right), w_{1}=\left(u_{1}, u_{1}\left(a_{2}\right), u_{1}\left(a_{1}\right)\right),\right. \\
\left.w_{i}=\left(u_{i}, u_{i}\left(a_{i+1}\right)\right), i=2, \ldots, m, \bar{u} \in \widehat{H}_{0}\right\} .
\end{gathered}
$$

We also define the spaces

$$
\begin{gathered}
H_{1 / 2}=\left\{\bar{u}, \bar{u}=\left(u_{1}, \ldots, u_{m}\right), u_{i} \in W_{2}^{1}\left(a_{i}, a_{i+1}\right), i=1, \ldots, m\right\}, \\
\widehat{H}_{1 / 2}=\left\{\bar{u}, \bar{u}=\left(u_{1}, \ldots, u_{m}\right), u_{i} \in W_{2}^{1}\left(a_{i}, a_{i+1}\right), i=1, \ldots, m,\right. \\
\left.u_{j}\left(a_{j+1}\right)=u_{j+1}\left(a_{j+1}\right), j=1, \ldots, m-1\right\} .
\end{gathered}
$$

Statement 3.2. $\mathscr{L}_{1}$ is dense in

$$
\mathscr{H}=\left(L_{2}\left(a_{1}, b_{1}\right) \oplus \mathbb{C} \oplus \mathbb{C}\right) \oplus \bigoplus_{i=2}^{m}\left(L_{2}\left(a_{i}, b_{i}\right) \oplus \mathbb{C}\right)
$$


Proof. Assume that $\left(u_{1}, \alpha_{1}, \alpha_{0}, u_{2}, \alpha_{2}, \ldots, u_{m}, \alpha_{m}\right) \in \mathscr{H}$. Consider the following functions:

$$
u_{i}^{0}(x)=\frac{a_{i+1}-x}{a_{i+1}-a_{i}} \alpha_{i-1}+\frac{x-a_{i}}{a_{i+1}-a_{i}} \alpha_{i}, \quad x \in\left[a_{i}, a_{i+1}\right], i=1, \ldots, m .
$$

From definitions of $u_{i}^{0}(x), i=1, \ldots, m$, we can see that

$$
u_{i}^{0}\left(a_{i+1}\right)=u_{i+1}^{0}\left(a_{i+1}\right)=\alpha_{i}, \quad i=1,2, \ldots, m-1 .
$$

Let $\bar{u}=\left(u_{1}, \ldots, u_{m}\right) \in \widehat{H}$. Consider the function

$$
z=\left(z_{1}, \ldots, z_{m}\right)=\left(u_{1}-u_{1}^{0}, \ldots, u_{m}-u_{m}^{0}\right)
$$

It is obvious that $z \in \bigoplus_{i=1}^{m} L_{2}\left(a_{i}, a_{i+1}\right)$. On the other hand, $\overline{\bigoplus_{i=1}^{m} \Phi\left(a_{i}, a_{i+1}, a_{i+1}\right)}=$ $\bigoplus_{i=1}^{m} L_{2}\left(a_{i}, a_{i+1}\right)$, where $\Phi\left(a_{i}, a_{i+1}\right)(i=1, \ldots, m)$ is a space of infinitely differentiable finite functions. Therefore, for an arbitrary $\varepsilon>0$, there exist the functions $h_{i} \in \Phi\left(a_{i}, a_{i+1}\right)$, $i=1, \ldots, m$, such that

$$
\sum_{i=1}^{m}\left\|z_{i}-h_{i}\right\|<\varepsilon
$$

By denoting $\tilde{h}_{i}=u_{i}^{0}+h_{i}$ from (3.17), we get

$$
\sum_{i=1}^{m}\left\|u_{i}-\tilde{h}_{i}\right\|_{L_{2}\left(a_{i}, a_{i+1}\right)}<\varepsilon,
$$

where $\tilde{h}_{i} \in C^{\infty}\left[a_{i}, a_{i+1}\right], \tilde{h}_{i}\left(a_{i}\right)=\alpha_{i-1}, i=1, \ldots, m$.

Thus,

$$
\|\left(u_{1}, u_{1}\left(a_{2}\right), u_{1}\left(a_{1}\right), u_{2}, u_{2}\left(a_{2}\right), \ldots, u_{m}, u_{m}\left(a_{m+1}\right)-\left(h_{1}, \alpha_{1}, \alpha_{0}, h_{2}, \alpha_{1}, \ldots, h_{m}, \alpha_{m}\right) \|_{\mathscr{\ell}}<\varepsilon\right.
$$

The following statement is proved in the same way.

Statement 3.3. $\widehat{H}_{0}$ is dense $\widehat{H}_{1 / 2}$.

Now, we prove that the condition (vi) holds. 
Let $\bar{u}=\left(u_{1}, \ldots, u_{m}\right), \bar{v}=\left(v_{1}, \ldots, v_{m}\right) \in \widehat{H}_{0}$, then

$$
\begin{aligned}
& \sum_{i=1}^{m}\left[\left(A_{i}(t) u_{i}, v_{i}\right)_{H^{i}(t)}+\sum_{v=1}^{s_{i}}\left(\sum_{k=1}^{m} C_{k v}^{i}(t) u_{k}, B_{i v} v_{i}\right)_{X_{v}^{i}(t)}\right] \\
& =\sum_{i=1}^{m}\left[-\int_{a_{i}}^{a_{i+1}} u_{i}^{\prime \prime} v_{i} d x+\left(-u_{1}^{\prime}\left(a_{1}\right), v_{1}\left(a_{1}\right)\right)\right. \\
& \left.\quad+\sum_{i=1}^{m}\left(u_{i}^{\prime}\left(a_{i+1}\right)-u_{i+1}^{\prime}\left(a_{i+1}\right), v_{i}\left(a_{i+1}\right)\right)+\left(u_{m}^{\prime}\left(a_{m+1}\right), v_{m}\left(a_{m}\right)\right)\right] \\
& =\sum_{i=1}^{m}\left[\left(u_{i}^{\prime}\left(a_{i}\right), v_{i}\left(a_{i}\right)\right)-\left(u_{i}^{\prime}\left(a_{i+1}\right), v_{i}\left(a_{i+1}\right)\right)\right] \\
& \quad-\sum_{i=1}^{m} \int_{a_{i}}^{a_{i+1}} u_{i}^{\prime} v_{i}^{\prime} d x-\left(u_{1}^{\prime}\left(a_{1}\right), v_{1}\left(a_{1}\right)\right)+\sum_{i=1}^{m-1} u_{i}^{\prime}\left(a_{i+1}\right) v_{i}^{\prime}\left(a_{i+1}\right) \\
& \quad-\sum_{i=1}^{m-1} u_{i+1}^{\prime}\left(a_{i+1}\right) v_{i}\left(a_{i+1}\right)+u_{m}^{\prime}\left(a_{m+1}\right) v_{m}\left(a_{m+1}\right)
\end{aligned}
$$$$
=\sum_{i=1}^{m}\left(u_{i}^{\prime}\left(a_{i}\right) v_{i}\left(a_{i}\right)-u_{i}^{\prime}\left(a_{i+1}\right) v_{i}^{\prime}\left(a_{i+1}\right)\right)-u_{1}^{\prime}\left(a_{1}\right) v_{1}\left(a_{1}\right)+\sum_{i=1}^{m-1} u_{i}^{\prime}\left(a_{i}\right) v_{i}\left(a_{i}\right)-\sum_{i=2}^{m} u_{i}^{\prime}\left(a_{i}\right) v_{i}\left(a_{i}\right)
$$$$
+u_{m}^{\prime}\left(a_{m+1}\right) v_{m}\left(a_{m+1}\right)+\sum_{i=1}^{m} \int_{a_{i}}^{a_{i+1}} u_{i}^{\prime} v_{i}^{\prime} d x=\sum_{i=1}^{m} \int_{a_{i}}^{a_{i+1}} u_{i}^{\prime} v_{i}^{\prime} d x .
$$

Similary, we obtain the following identity:

$$
\sum_{i=1}^{m}\left[\left(u_{i}, A_{i}(t) v_{i}\right)_{H^{i}(t)}+\sum_{v=1}^{s_{i}}\left(B_{i v} v_{i}, \sum_{k=1}^{m} C_{k v}^{i}(t) u_{k}\right)_{X_{v}^{i}(t)}\right]=\sum_{i=1}^{m} \int_{a_{i}}^{a_{i+1}} u_{i}^{\prime} v_{i}^{\prime} d x .
$$

Thus, by virtue of (3.20)-(3.21), the condition (vi) holds.

From (3.20) or (3.21), putting $v_{i}=u_{i}$, we also obtain the identity

$$
\sum_{i=1}^{m} \int_{a_{i}}^{a_{i+1}} u_{i}^{2^{\prime}} d x=\sum_{i=1}^{m}\left[\left(A_{i}(t) u_{i}, u_{i}\right)_{H^{i}(t)}+\sum_{v=1}^{s_{i}}\left(\sum_{k=1}^{m} C_{k v}^{i}(t) u_{k}, B_{i v} u_{i}\right)_{X_{v}^{i}(t)}\right],
$$

that is, condition (viii) is satisfied, $c_{1}=c_{2}=1$. 
Now, we verify fulfillment of condition (ix). To that end, we consider the mixed problem

$$
\begin{gathered}
\lambda u_{i}-p_{i}(t) u_{i}^{\prime \prime}=h_{i}(x), \quad i=1,2, \ldots, m, \\
\lambda u_{1}\left(a_{1}\right)-q_{0}(t) u_{1}^{\prime}\left(a_{1}\right)=h_{10}, \\
\lambda u_{i}\left(a_{i+1}\right)+q_{i}(t)\left[u_{i}^{\prime}\left(a_{i+1}\right)-u_{i+1}^{\prime}\left(a_{i+1}\right)\right]=h_{i 0}, \quad i=1,2, \ldots, m-1, \\
\lambda u_{m}\left(a_{m+1}\right)+q_{m}(t) u_{m}^{\prime}\left(a_{m+1}\right)=h_{m 0},
\end{gathered}
$$

where $h_{i} \in L_{2}\left(a_{i}, a_{i+1}\right), i=1, \ldots, m ; h_{j 0} \in R, j=0,1, \ldots, m, \lambda \in R$.

Let $h_{i}(x)$ be the extend of function $h_{i}(x)$ to $R$. We consider the system of the differential equations

$$
\lambda \tilde{u}_{i}-p_{i}(t) \tilde{u}_{i_{x x}}=\tilde{h}_{i}(x), \quad i=1,2, \ldots, m .
$$

Hence, we have

$$
\lambda \widehat{\tilde{u}}_{i}-k^{2} p_{i}(t) \widehat{\widetilde{u}}_{i_{x x}}=\widehat{\widetilde{h}}_{i}(x), \quad i=1,2, \ldots, m,
$$

where $\hat{g}=F[g]$ is a Fourier transformation of the function $g(x)$. From (3.26), we obtain $\widehat{\widetilde{u}}=\widehat{\widetilde{h}}_{i} /\left(\lambda+k^{2} p_{i}(t)\right)$, then functions $\tilde{u}_{i}=F^{-1}\left[\widehat{\widetilde{u}}_{i}\right]=F^{-1}\left[\widehat{\tilde{h}}_{i} /\left(\lambda+k^{2} p_{i}(t)\right)\right]$ satisfy (3.25), and their constrictions on $\left(a_{i}, a_{i+1}\right)$ satisfy the (3.23). It is clear that $\tilde{u}_{i} \in W_{2}^{2}\left(a_{i}, a_{i+1}\right)$. Considering linearity of the problem (3.23), (3.24), the solution can be represented in the form

$$
u_{i}=v_{i}+\tilde{u}_{i}
$$

where $v_{i}=u_{i}-\tilde{u}_{i}$ is a solution of the following problem:

$$
\begin{gathered}
\lambda v_{i}(x)-p_{i}(t) v_{i}^{\prime \prime}(x)=0, \\
\lambda v_{1}\left(a_{1}\right)-q_{0}(t) v_{1}^{\prime}\left(a_{1}\right)=\tilde{h}_{10}, \\
\lambda v_{i}\left(a_{i+1}\right)+q_{i}(t)\left[v_{i}^{\prime}\left(a_{i+1}\right)-v_{i+1}^{\prime}\left(a_{i+1}\right)\right]=\widetilde{h}_{i 0}, \quad i=1,2, \ldots, m-1, \\
\lambda v_{m}\left(a_{m+1}\right)+q_{m}(t) v_{m}^{\prime}\left(a_{m+1}\right)=\tilde{h}_{m 0},
\end{gathered}
$$

where $\tilde{h}_{10}=h_{10}-\lambda \tilde{u}_{1}\left(a_{1}\right)+q_{0}(t) \widetilde{u}_{1}^{\prime}\left(a_{1}\right)$,

$$
\begin{gathered}
\tilde{h}_{i 0}=h_{i 0}-\lambda \tilde{u}_{i}\left(a_{i+1}\right)-q_{i}(t)\left[\tilde{u}_{i}^{\prime}\left(a_{i+1}\right)-\tilde{u}_{i+1}^{\prime}\left(a_{i+1}\right)\right], \quad i=1,2, \ldots, m-1, \\
\tilde{h}_{m 0}(0)=h_{m_{0}}-\lambda \tilde{u}_{m}\left(a_{m+1}\right)+q_{m}(t) \tilde{u}_{m}^{\prime}\left(a_{m+1}\right) .
\end{gathered}
$$


A general solution of a system (3.28) is found in the following form:

$$
v_{i}(x)=c_{i 1} e^{-\left(x-a_{i}\right) \sqrt{\lambda / p_{i}(t)}}+c_{i 2} e^{-\left(b_{i}-x\right) \sqrt{\lambda / p_{i}(t)}}, \quad i=1,2, \ldots, m
$$

Then, for determination of $c_{i 1}, c_{i 2}, i=1,2, \ldots, m$, from (3.29), we get the following system of the algebraic equations:

$$
\begin{aligned}
& \lambda\left(c_{11}+c_{12} e^{-\left(a_{2}-a_{1}\right) \sqrt{\lambda / p_{i}(t)}}\right)-q_{0}(t) \sqrt{\frac{\lambda}{p_{i}(t)}}\left(c_{11}-c_{12} e^{-\left(a_{2}-a_{1}\right) \sqrt{\lambda / p_{i}(t)}}\right)=\tilde{h}_{0} \\
& \lambda\left(c_{i 1} e^{-\left(a_{i+1}-a_{i}\right) \sqrt{\lambda / p_{i}(t)}}-c_{i 2}\right)+q_{i}(t) \sqrt{\frac{\lambda}{p_{i}(t)}}\left(c_{i 1} e^{-\left(a_{i+1}-a_{i}\right) \sqrt{\lambda / p_{i}(t)}}+c_{i 2}\right) \\
& -\sqrt{\frac{\lambda}{p_{i+1}(t)}}\left(c_{i+1,1}+c_{i+1,2} e^{-\left(a_{i+2}-a_{i+1}\right)} \sqrt{\lambda / p_{i+1}(t)}\right)=\tilde{h}_{i 0}, \quad i=1,2, \ldots, m-1, \\
& c_{i 1} e^{-\left(a_{i+1}-a_{i}\right) \sqrt{\lambda / p_{i}(t)}}-c_{i 2}-\left(c_{i+1,1}-c_{i+1,2} e^{-\left(a_{i+2}-a_{i+1}\right) \sqrt{\lambda / p_{i+1}(t)}}\right)=0, \quad i=1, \ldots, m-1, \\
& \lambda\left(c_{m 1} e^{-\left(a_{m+1}-a_{m}\right) \sqrt{\lambda / p_{m}(t)}}+c_{m 2}\right)+\left(-c_{m 1} e^{-\left(a_{m+1}-a_{m}\right) \sqrt{\lambda / p_{m}(t)}}+c_{m 2}\right)=\tilde{h}_{m_{0}} .
\end{aligned}
$$

Let $R(\lambda)$ be a matrix of coefficients of system (3.32). From (3.32), it is clear that $R(\lambda)=$ $R_{0}(\lambda)+R_{1}(\lambda)$, where $\operatorname{det} R_{0}(\lambda) \rightarrow+\infty$ and $\operatorname{det} R_{1}(\lambda) \rightarrow 0$ as $\lambda \rightarrow+\infty$. Thus, for sufficiently large $\lambda, R(\lambda)$ is invertible and det $R(\lambda) \rightarrow+\infty$. Therefore, the system (3.32) has a unique solution.

Thus, for sufficiently positive large $\lambda$, the problem (3.23)-(3.24) has a unique solution $u=\left(u_{1}, \ldots, u_{m}\right) \in H_{0}$. $\left(1^{0}\right)-\left(6^{0}\right)$.

Thus, the condition (ix) is satisfied. The fulfillment of other conditions follows from

Now, let us consider a class of nonlinear equations, for which the large solvability theorem takes place.

Let

$$
\begin{gathered}
f_{i}\left(t, x, u_{i}, u_{i}^{\prime}, \dot{u}_{i}, \varphi(\bar{u}, \dot{\bar{u}})\right)=-\left|u_{i}\right|^{\rho_{i}} u_{i}+f_{1 i}\left(t, x, u_{i}, u_{i}^{\prime}, \dot{u}_{i}, \tilde{\varphi}_{i}(\bar{u}, \dot{\bar{u}})\right), \\
g_{0}\left(t, \psi_{0}(\bar{u}, \dot{\bar{u}})\right)=-\left|u_{1}\left(a_{1}\right)\right|^{\tau_{0}} u_{1}\left(a_{1}\right)+g_{01}\left(t, \psi_{0}(\bar{u}, \dot{\bar{u}})\right), \\
g_{i}\left(t, \psi_{i}(\bar{u}, \dot{\bar{u}})\right)=-\left|u_{i}\left(a_{i+1}\right)\right|^{\tau_{i}} u_{i}\left(a_{i+1}\right)+g_{i 1}\left(t, \tilde{\psi}_{i}(\bar{u}, \dot{\bar{u}})\right), \quad i=1,2, \ldots, m,
\end{gathered}
$$

where $\rho_{i} \geq 0, \tau_{j} \geq 0, i=1,2, \ldots, m ; j=0,1, \ldots, m$ and

$\left(7^{0}\right) f_{1 i}, g_{1 j}, \widetilde{\varphi}_{i}$ and $\widetilde{\psi}_{j}, i=1,2, \ldots, m, j=1,2, \ldots, m$ satisfy the conditions $\left(2^{0}\right)--\left(5^{0}\right)$.

$\left(8^{0}\right)\left|f_{i}\left(t, x, u_{i}, v_{i}, \xi_{i}, \eta\right)\right| \leq c\left(1+\left|u_{i}\right|^{\left(\rho_{i}+2\right) / 2}+\left|v_{i}\right|+\left|\xi_{i}\right|+|\eta|\right)$, 


$$
\begin{aligned}
\left(9^{0}\right)\left|g_{0 i}(t, \eta)\right| & \leq c(1+|\eta|), \\
\left(10^{0}\right)\left|\varphi_{i}(\bar{u}, \bar{v})\right| & \leq c\left(1+\sum_{i=1}^{n}\left|u_{i}\right|^{\left(\rho_{i}+2\right) / 2}+\left|v_{i}\right|^{2}+\left|u_{i}\left(y_{i}\right)\right|^{\left(\tau_{i}+2\right) / 2}\right),
\end{aligned}
$$

where $y_{i}=a_{i+1}, i=0,1, \ldots, m, \rho=\max \left(\min _{i=1,2, \ldots, m}\left(\rho_{i}, 2\right)\right)$.

Theorem 3.4. Let conditions $\left(7^{0}\right)-\left(10^{0}\right)$ be held and initial data satisfy the condition $\left(6^{0}\right)$, then the problem (3.1) has a unique solution $\bar{u}=\left(u_{1}, \ldots, u_{m}\right)$, where

$$
\begin{gathered}
u_{i} \in C^{2}\left([0, T] ; L_{2}\left(a_{i}, a_{i+1}\right)\right) \cap C^{1}\left([0, T] ; W_{2}^{1}\left(a_{i}, a_{i+1}\right)\right) \cap C\left([0, T] ; W_{2}^{2}\left(a_{i}, a_{i+1}\right)\right), \\
u_{i}\left(t, a_{i}\right), u_{i}\left(t, a_{i+1}\right) \in C^{2}([0, T] ; R), \quad i=1,2, \ldots, m .
\end{gathered}
$$

\section{References}

[1] Y. Yakubov, "Hyperbolic differential-operator equations on a whole axis," Abstract and Applied Analysis, no. 2, pp. 99-113, 2004.

[2] P. Lancaster, A. Shkalikov, and Q. Ye, "Strongly definitizable linear pencils in Hilbert space," Integral Equations and Operator Theory, vol. 17, no. 3, pp. 338-360, 1993.

[3] J.-L. Lions and E. Magenes, Problemes aux Limites non Homogenes et Applications, vol. 1, Dunod, Paris, France, 1968.

[4] M. Reed and B. Simon, Methods of Modern Mathematical Physics. II. Fourier Analysis, Self-Adjointness, Academic Press, New York, NY, USA, 1975.

[5] T. Kato, "Linear evolution equations of "hyperbolic" type. II," Journal of the Mathematical Society of Japan, vol. 25, pp. 648-666, 1973.

[6] T. J. R. Hughes, T. Kato, and J. E. Marsden, “Well-posed quasi-linear second-order hyperbolic systems with applications to nonlinear elastodynamics and general relativity," Archive for Rational Mechanics and Analysis, vol. 63, no. 3, pp. 273-294, 1977.

[7] S. Yakubov and Y. Yakubov, Differential-Operator Equations. Ordinary and Partial Differential Equations, vol. 103 of Chapman \& Hall/CRC Monographs and Surveys in Pure and Applied Mathematics, Chapman \& Hall/CRC, Boca Raton, Fla, USA, 2000.

[8] S. Q. Krein, Linear Differential Equation in Banach Spaces, Nauka, Moscow, Russia, 1967.

[9] A. B. Aliev, "Solvability "in the large" of the Cauchy problem for quasilinear equations of hyperbolic type," Doklady Akademii Nauk SSSR, vol. 240, no. 2, pp. 249-252, 1978.

[10] A. M. Samollenko and N. A. Perestyuk, Impulsive Differential Equations, vol. 14 of World Scientific Series on Nonlinear Science. Series A: Monographs and Treatises, World Scientific, River Edge, NJ, USA, 1995. 